\section{BMJ Open Respiratory Research}

\title{
Feasibility study of in vitro drug sensitivity assay of advanced non-small cell lung adenocarcinomas
}

Emoke Papp, ${ }^{1}$ Anita Steib, ${ }^{2}$ Elhusseiny MM Abdelwahab, ${ }^{3,4}$ Judit Meggyes-Rapp, ${ }^{2,3}$ Laszlo Jakab, ${ }^{5}$ Gabor Smuk, ${ }^{6}$ Erzsebet Schlegl, ${ }^{7}$ Judit Moldvay, ${ }^{7,8}$ Veronika Sárosi, ${ }^{1}$ Judit E Pongracz (D) ${ }^{3,4}$

\section{ABSTRACT}

Background Despite improved screening techniques, diagnosis of lung cancer is often late and its prognosis is poor. In the present study, in vitro chemosensitivity of solid tumours and pleural effusions of lung adenocarcinomas were analysed and compared with clinical drug response. Methods Tumour cells were isolated from resected solid tumours or pleural effusions, and cryopreserved. Three-dimensional (3D) tissue aggregate cultures were set up when the oncoteam reached therapy decision for individual patients. The aggregates were then treated with the selected drug or drug combination and in vitro chemosensitivity was tested individually measuring ATP levels. The clinical response to therapy was assessed by standard clinical evaluation over an 18 months period. Results Based on the data, the in vitro chemosensitivity test results correlate well with clinical treatment response. Conclusions Such tests if implemented into the clinical decision making process might allow the selection of an even more individualised chemotherapy protocol which could lead to better therapy response.

\section{INTRODUCTION}

While the recently improved treatment strategies have resulted in better survival statistics in many cancers, in non-small cell lung cancer (NSCLC) the 1-year overall survival at a locally advanced or metastatic stage barely exceeds 20\%. Although key mutations aid clinical decision-making and facilitate the application of targeted therapies, radical improvements have not been observed and the 5-year survival rate remains at approximately $5 \%$. $^{1}$ Although next-generation sequencing has become a cornerstone of therapy guidance, ${ }^{2}$ clinical decision-making remains difficult due to the histological diversity of NSCLC (adeno, squamosous, large cell) and the variation of the mutation characteristics of the different subtypes. $^{3}$ Based on clinical guidelines, patients with advanced lung adenocarcinoma (AC) are tested for the presence of Kirsten rat sarcoma 2 viral oncogene homolog (KRAS) and epidermal growth factor receptor (EGFR) mutations and rearrangements involving anaplastic lymphoma kinase (ALK). ${ }^{4}$ Unfortunately, analysis of tumour mutations can only describe the mutations existing at the specific location where the sample was taken from and at the time of sample taking. By the time therapy is selected, additional mutations may have occurred. ${ }^{56}$ Due to the diversity of histology as well as mutations in NSCLC, the first-line treatment of locally advanced or inoperable cancer is platinum based, which can in itself dramatically increase the mutation rate. ${ }^{7}$ The relatively slow acting immunotherapies are only considered as an alternative in specific cases ${ }^{8}$; therefore, chemotherapy remains the principal treatment modality in advanced NSCLC. To improve chemotherapy response rates, drug sensitivity assays have been under intense investigation. ${ }^{9}$ It was recognised that tissues derived from the original tumour represent the tumour composition suitably well to test chemosensitivity on freshly isolated tumour cells in vitro. ${ }^{10}$ Most published tests, however, have not been performed on advanced NSCLC. ${ }^{9}$ While the statistical analysis of data in the current literature involving different tumours looks convincing, clinicians remain wary of such tests due to the clinical complexity of individual treatment responses. In the present study, we have performed in vitro drug sensitivity analysis in advanced NSCLC AC samples, to investigate sensitivity to currently recommended drugs and compared the results to clinical therapy response.

\section{MATERIALS AND METHODS}

Resected solid tissue samples were digested using a Miltenyi Tumor Dissociation Kit (Miltenyi Biotec, Auburn, USA). Cells from pleural fluid were centrifuged $(600 \mathrm{~g}, 10 \mathrm{~min})$, then isolated by Ficol separation. Red blood cells were removed by Red Blood Cell Lysis Buffer (Roche, Mannheim, Germany). Cells 
were cryopreserved using Cryo-SFM (PromoCell, Heidelberg, Germany) and stored at $-80^{\circ} \mathrm{C}$ until used.

Cryopreserved tumour cells were combined with normal human lung fibroblast cells (1:1) and then aggregated in a low-attachment 96-well plate (Corning, New York, USA). ${ }^{10}$

Tissue aggregates were treated with chemotherapeutic compounds (Selleckem, Munich, Germany) selected by the oncoteam.

Concentrations for in vitro treatments were based on the literature ${ }^{1112}$ and in vitro concentration tests performed in our laboratory. Drug concentrations were as follows: cisplatin $(7 \mu \mathrm{M}),{ }^{13}{ }^{14}$ carboplatin $(\mathrm{CBP} ; 100 \mu \mathrm{M}),{ }^{14}$ vinorelbine $(150 \mathrm{nM}),{ }^{15}$ gemcitabine $(30 \mu \mathrm{M}),{ }^{16}$ paclitaxel $(100 \mathrm{nM}),{ }^{17}$ pemetrexed $(10 \mu \mathrm{M}),{ }^{16}$ erlotinib $(100 \mathrm{nM})^{18}$ and gefitinib $(100 \mathrm{nM}) .{ }^{19}$ Treatments were carried out for 48 hours at $37^{\circ} \mathrm{C}$ in $5 \% \mathrm{CO}_{2}$ atmosphere in four parallels.

In vitro viability assay was performed using the threedimensional (3D) CellTiter Glo (Promega, Madison, USA) kit, measured in a PerkinElmer Plate Reader (PerkinElmer, Waltham, USA).

CT, MRI, chest X-ray and abdominal ultrasound methods were used for Response Evaluation Criteria
In Solid Tumours (RECIST V.1.1) evaluations. $^{20}$ In the selected patient populations, only stable (SD) and progressive (PD) diseases were distinguished. The in vitro chemosensitivity results were compared with the patients' clinical responses to chemotherapy at 2-3 months and patients were monitored over 18 months. One-way analysis of variance was used for statistical analysis and $\mathrm{p}<0.05$ was considered as significant.

\section{RESULTS}

The study design and patient exclusion criteria are summarised in figures 1 and 2, respectively. Patient information is summarised in table 1 .

Cells from cancer tissue or pleural effusion (PE) samples obtained from each patient were limited; therefore, the in vitro chemosensitivity tests were performed based on the clinical decision for treatment. The cryopreserved samples were thawed (viability routinely exceeded $90 \%$; online supplementary S. figure 1), aggregates were made, then drug sensitivity tests were performed with drugs or drug combinations selected by the oncoteam (online supplementary S. figure 2). The in vitro viability

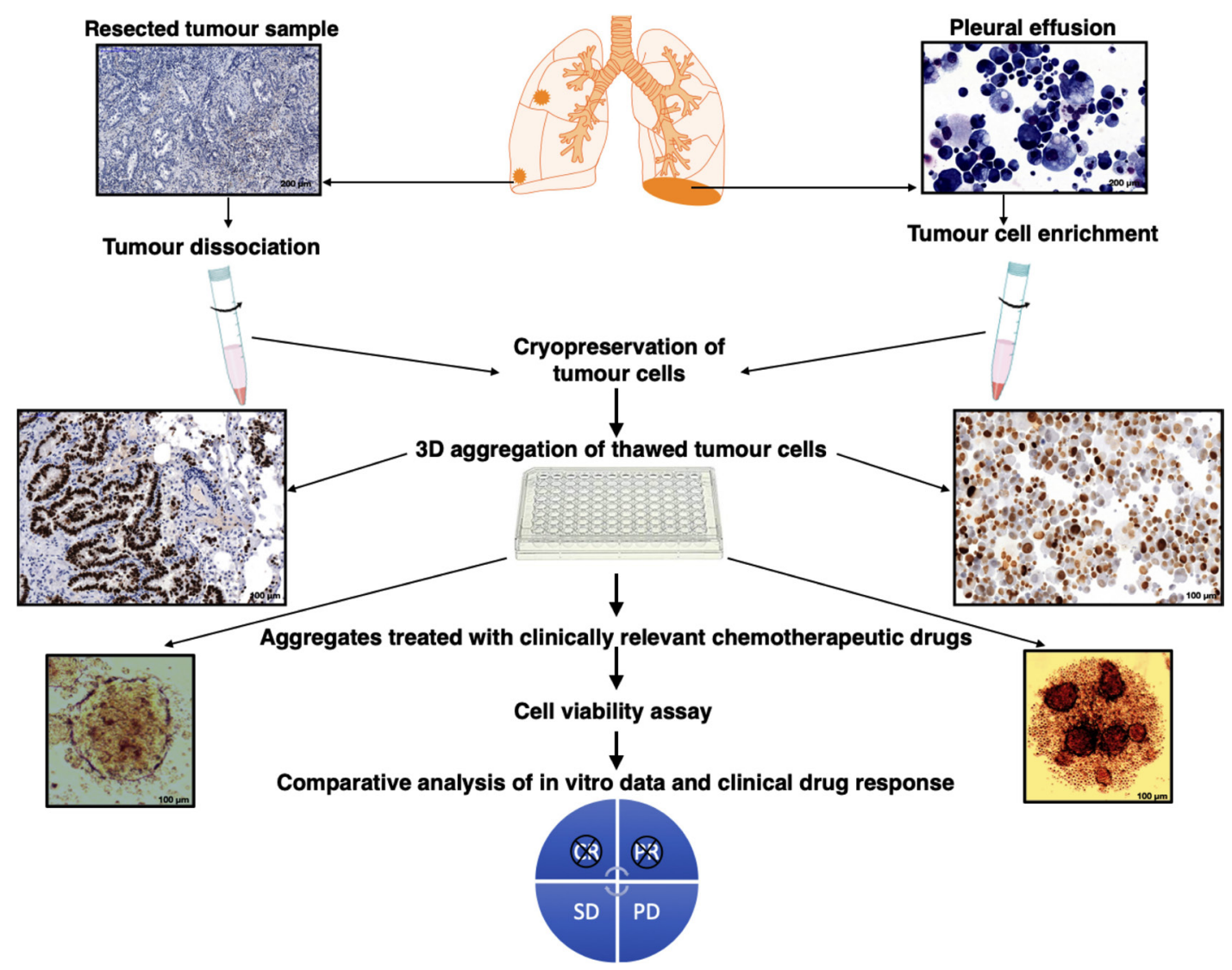

Figure 1 Study design. All the samples were freshly cryopreserved as single cell suspensions, then thawed when the oncoteam made a decision for therapy. Both the resected tissue and the tumour-enriched pleural effusion stained positive for TTF1 and both sample types were routinely tested for Kirsten rat sarcoma 2 viral oncogene homolog, epidermal growth factor receptor and anaplastic lymphoma kinase mutations. Single cell suspensions were cryopreserved and stored at $-80^{\circ} \mathrm{C}$ until used. Samples were thawed and placed into three-dimensional (3D) aggregate cultures, then treated with the corresponding chemotherapeutic agent(s) selected by the oncoteam. Patient therapy responses were monitored and compared with the in vitro assay results. PD, progressive disease; SD, stable disease; TFF1, Thyroid transcription factor 1. 


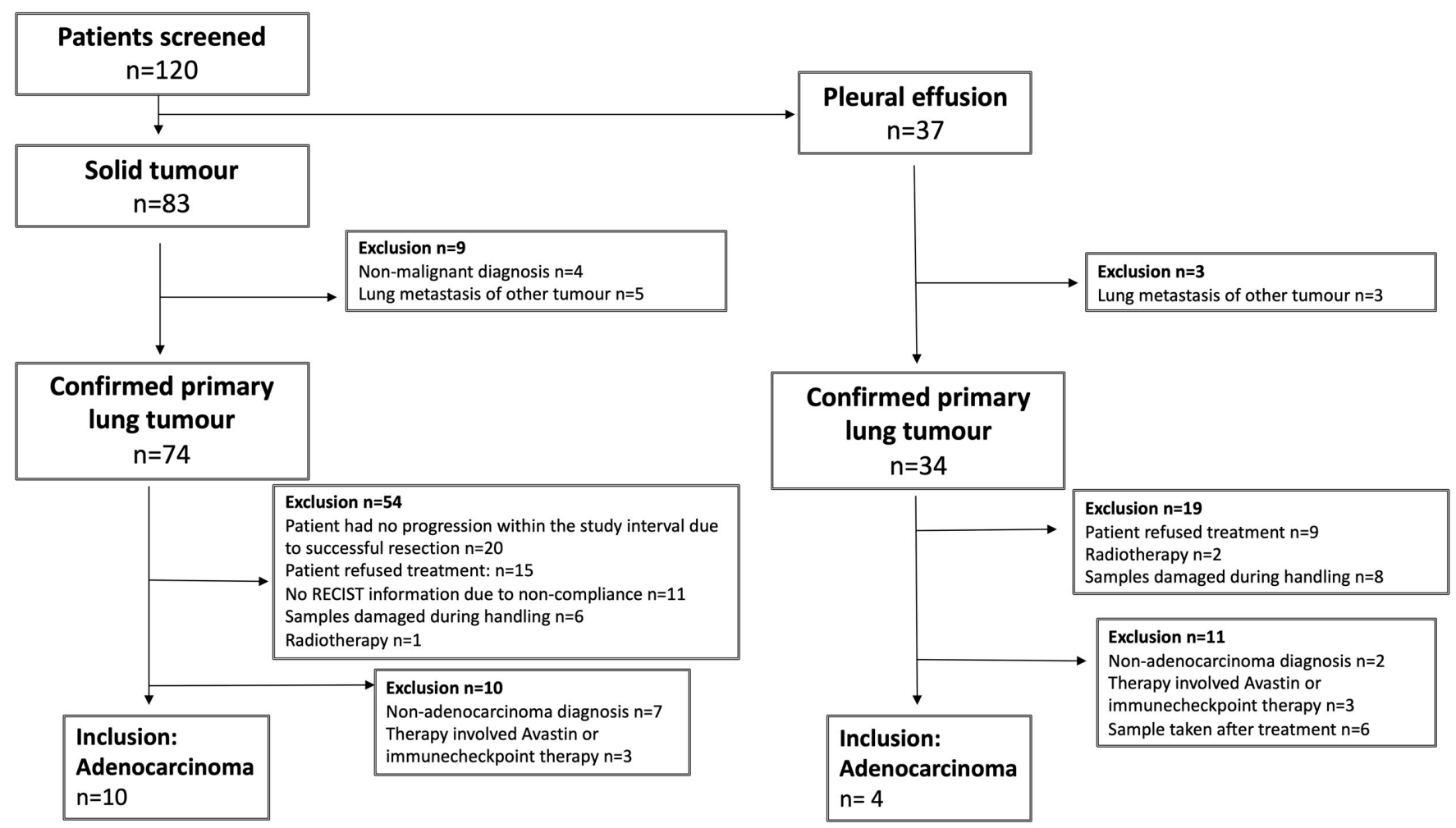

Figure 2 Exclusion criteria. Out of the 120 patients only 14 met the inclusion criteria, 10 for solid tumours and 4 for pleural effusions. The exclusion criteria are named in the boxes along with the number of patients excluded from the study. RECIST, Response Evaluation Criteria In Solid Tumours.

test results were compared with the RECIST 1.1 data. ${ }^{16}$ In vitro mean viability values at and below 0.8 (induction of cell death $0.2<$ ) corresponded to patients with clinically $\mathrm{SD}$, while a mean viability value of 0.9 and above (no or low level $(<0.1)$ induction of cell death) corresponded to patients with clinically PD (figure 3A; online supplementary table 1). Cut-off values are explained in online supplementary S. figure 3. Sample numbers on the figure correspond to the patient numbers in table 1 . The patient who donated the KRAS mutant solid tumour sample (S1) was PD during clinical observation, then following cisplatin+pemetrexed combination therapy became stable (SD). S1 patient became PD again after changing the treatment to pemetrexed monotherapy and the RECIST result correlated with the in vitro chemosensitivity analysis. The change in therapy was forced by severe adverse reactions to cisplatin. Patients S2 (wild type (WT)) and S9 (KRAS) responded well to cisplatin+vinorelbine combination as they were both SD at clinical examination and in vitro testing. Patients S4 (KRAS), S7 (KRAS), S8 (undisclosed mutation status) and S10 (EGFR) were SD correlating to the in vitro analysis. Patient samples S3 (KRAS), S5 (KRAS) and S6 (KRAS) remained firmly non-responsive to therapy and clinically PD after evaluation (figure $3 \mathrm{~A}$ ). The in vitro test results correlated well with the clinical data (figure 3A). Among the PE samples, donor of PE sample 2 (PE2) was initially SD after cisplatin+pemetrexed combination treatment but became PD when due to severe reactions to cisplatin, treatment was changed to pementrexed monotherapy (figure 3A). The same chemosensitivity response was detected also in vitro. Discrepancies between clinical and in vitro evaluation were detected in some cases. Correcting the corresponding in vitro data with the time course of progression information (figure 3B), a stronger association between the in vitro viability analysis and patient response to therapy (figure 3C) was detected.

To investigate the possibility whether the abovementioned in vitro drug sensitivity test could supplement the clinical decision-making process, a PE sample was selected for further studies. The patient who donated the sample did not respond to the clinically offered CBP-paclitaxel combination therapy (PD; figure 4). The in vitro chemosensitivity analysis using CBP-paclitaxel matched the clinical response (relative cell viability values were above 0.9 , no induction of cell death; figure 4 ). Another, clinically approved combination for therapy in this particular case could have been CBP-pemetrexed. In vitro analysis of the sample using $\mathrm{CBP}$-pemetrexed treatment of cell aggregates reduced cell viability below 0.8 (effective induction of cell death) that is in the $\mathrm{SD}$ range of the therapy response (figure 4).

\section{DISCUSSION}

According to the US Precision Medicine Initiative, ${ }^{21}$ the arsenal of precision medicine should be at the fingertips of every oncologist. The clinical reality, however, 


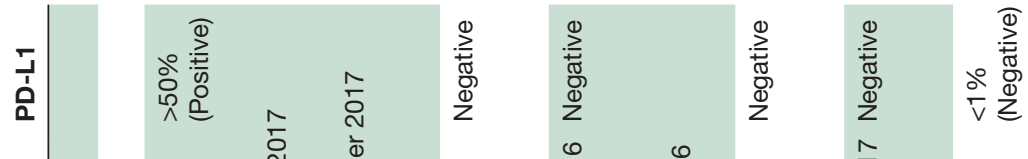

矛

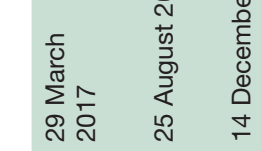
กั

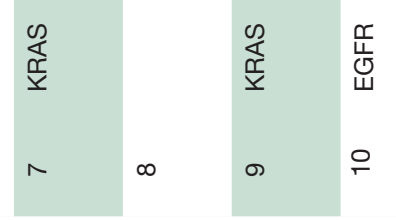




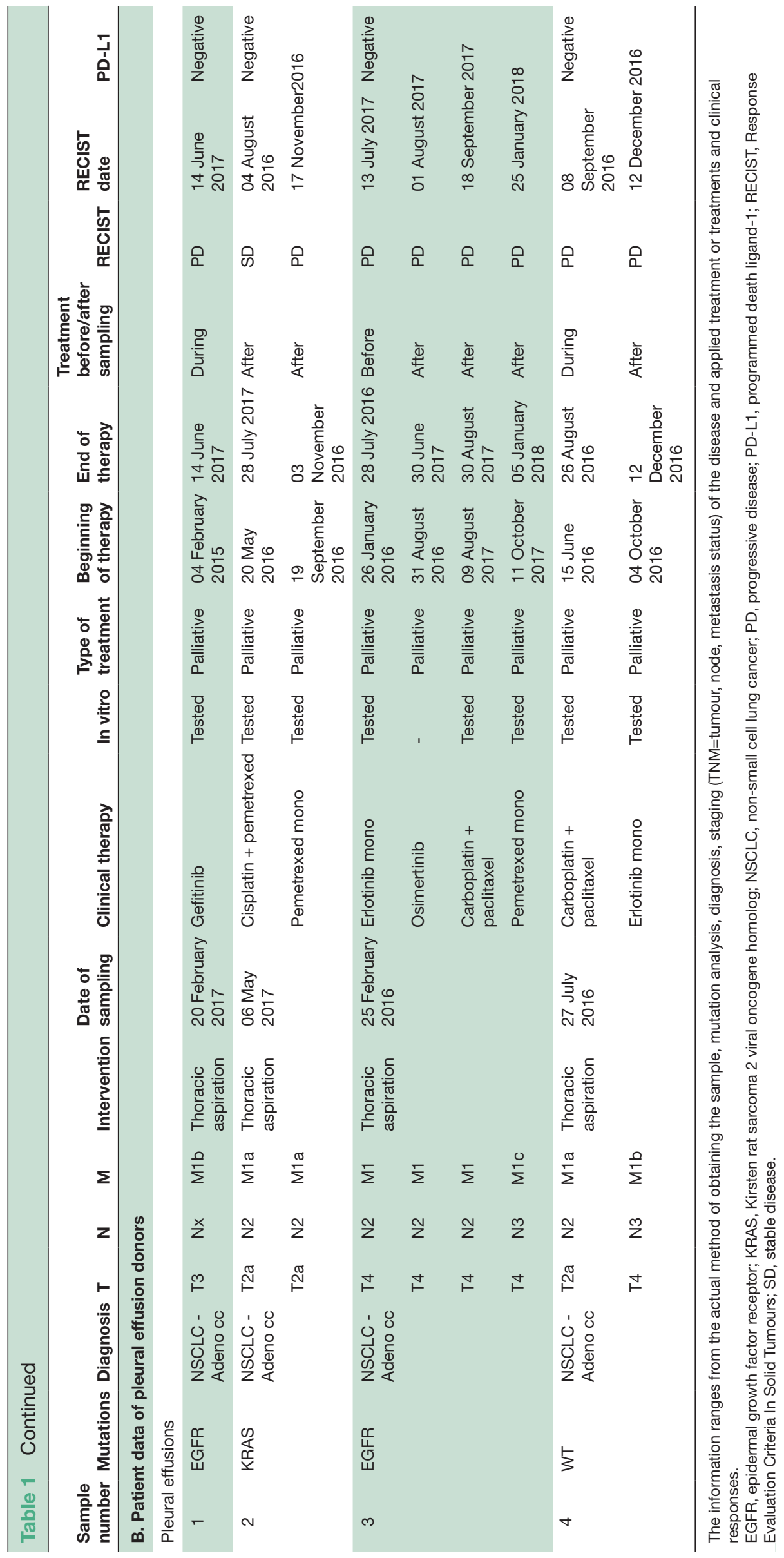




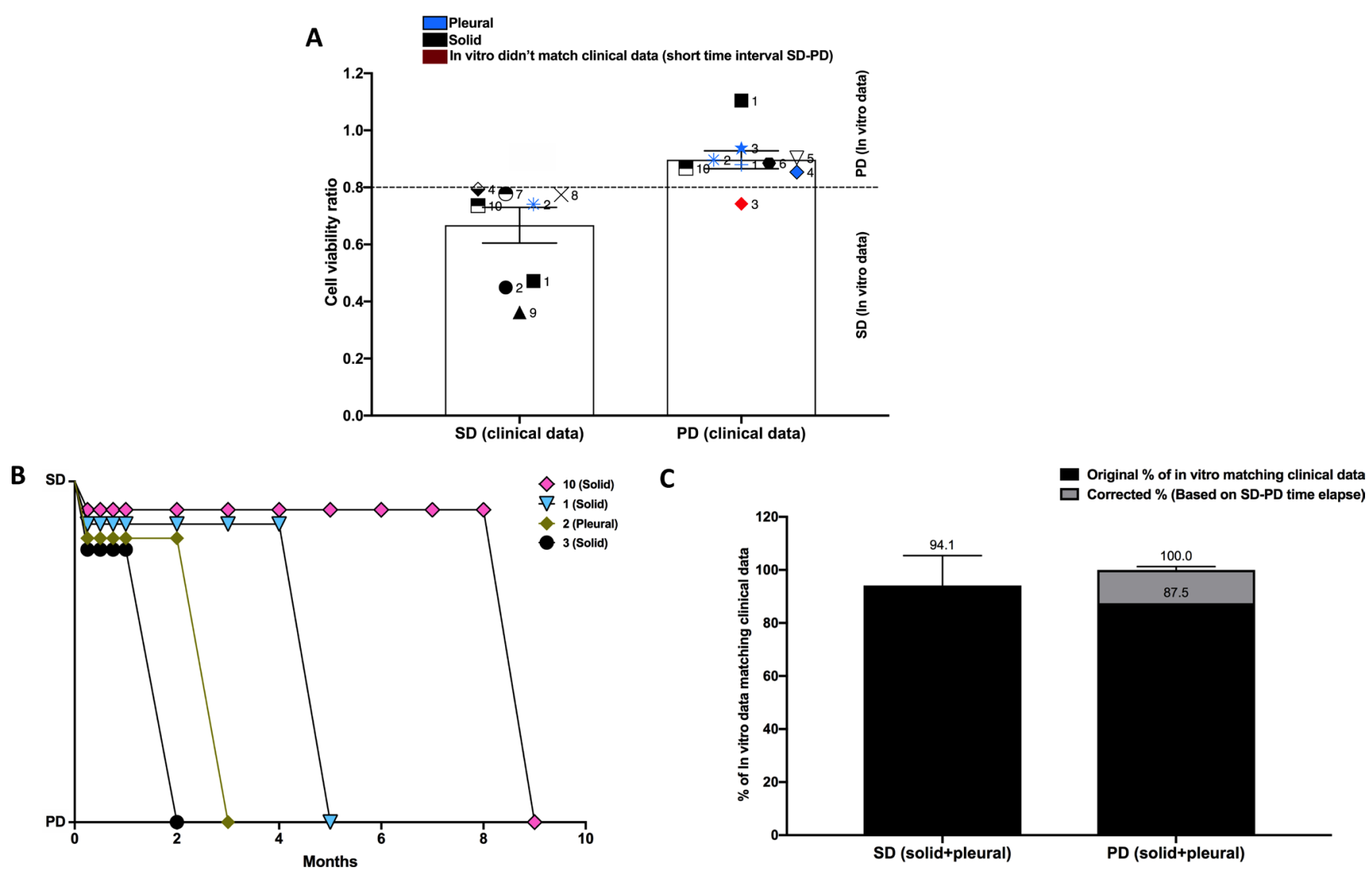

Figure 3 Chemosensitivity analysis. (A) Three-dimensional (3D) aggregate cultures were treated with patient-specific chemotherapeutic agents as determined by the oncoteam. After incubation at $37^{\circ} \mathrm{C}$ for 48 hours in a 96 -well plate, ATP levels corresponding to cell viability were determined using a 3D CellTiter Glo kit. In vitro viability data were compared with Response Evaluation Criteria In Solid Tumours (RECIST1.1) data when it became available. Patient-specific data are shown individually and marked with the patient identifying number used in the study. (B) Individual patient data shown in association of time laps to disease progression. (C) Percentage of correspondence between clinical RECIST1.1 information and in vitro analysis results. PD, progressive disease; SD, stable disease.

is different. Traditional chemotherapies are still the remaining treatment options for patients with stage III B or IV NSCLC tumours, that carry no targetable mutations, and are not positive for PD-L1. However, decisionmaking as chemosensitivity of the tumour is currently not tested routinely. Oncologists, who are bound by specific clinical guidelines, still need more than one treatment option to offer a patient. Even if the approved guidelines provide some choices, the fast progressing disease allows little time to select additional tests for the presence or absence of biomarkers indicating previously unpredicted therapeutic targets. The in vitro drug sensitivity analysis in the above study was performed to test whether the clinical effect of chemotherapeutic drug combinations could be tested using a simple and fast method where targeted therapy was not available for the patients. Compared with previous studies and test methods, ${ }^{22}$ we intentionally remained within the current routine clinical boundaries. Instead of using a vast number of mutation analyses ${ }^{23}$ and artificial intelligence ${ }^{24}$ to achieve better accuracy, we simply selected the patient population more carefully. For example, patients who were subjected to Avastin or immune checkpoint treatment were excluded from the test, as the test tissue was lacking blood supply and contained no immune cells. Such strict selection criteria had the consequence of a drastically reduced number of patients whose in vitro and clinical data were suitable for comparison. Although the number of patients were limited in the study, the in vitro data indicate that in vitro chemosensitivity tests could aid clinical drug selection and potentially expand survival even for patients with advanced lung AC. The fact that we are able to test more than one chemotherapy combination in vitro (figure 4) raises the possibility that an approved in vitro drug sensitivity test could make clinical decision-making easier. The in vitro process is feasible and could be easily added to the decision-making process. Partly, because it does not require additional sample taking from the patient and does not increase the workload of clinical staff.

In conclusion, chemosensitivity tests could supplement the clinical diagnostic arsenal as: (1) the assays can be performed from a small number of cells (1000 cell/well); therefore, even samples from distinct metastatic sites can be tested if sample taking is clinically feasible. (2) Cryopreservation of tumour cells allows sufficient time to perform additional diagnostic tests. (3) The analysis 


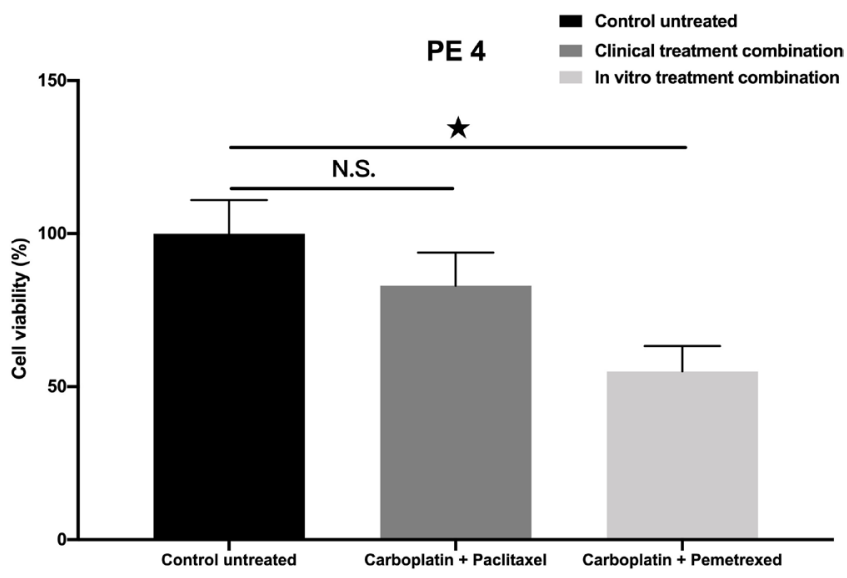

Figure 4 Testing optional drug sensitivity. Clinical application of carboplatin-paclitaxel combination therapy resulted in progressive disease with a matching in vitro chemosensitivity analysis of cell viability values above 0.9. Treatment of tissue culture with carboplatin-pemetrexed in vitro reduced cell viability below 0.8 that is in the stable disease range of therapy response. Viability compared with the untreated control was significantly lower when cell cultures were incubated with carboplatin-pemetrexed combination $(p<0.01)$. PE, pleural effusion; N.S., not significant.

provides information within 48 hours, which is vital for patients with fast progressing tumours.

Additionally, the above system could also be introduced into drug development. To reduce systemic toxicity, novel prodrug systems are being developed..$^{25}$ Although the $3 \mathrm{D}$ tissue aggregate is not suitable to test most prodrugs, the toxic effects of the active metabolite can be tested in the above system.

The prediction of the clinical response to chemotherapeutic drugs remains a major challenge in clinical oncology. If our simple and fast in vitro method were to be used to test chemosensitivity and if that test result is added to the patient's full clinical assessment, a decision for therapy could be made with increased confidence.

\author{
Author affiliations \\ ${ }^{1}$ Internal Medicine, Pulmonology, The Medical School and Clinical Centre, \\ University of Pecs, Pecs, Baranya, Hungary \\ ${ }^{2}$ Research, Humeltis Ltd, Pecs, Baranya, Hungary \\ ${ }^{3}$ Pharmaceutical Biotechnology, Faculty of Pharmacy, University of Pecs, \\ Pecs, Baranya, Hungary \\ ${ }^{4}$ Szentagothai Research Centre, University of Pecs, Pecs, Baranya, Hungary \\ ${ }^{5}$ Surgery, The Medical School and Clinical Centre, University of Pecs, Pecs, \\ Baranya, Hungary \\ ${ }^{6}$ Pathology, The Medical School and Clinical Centre, University of Pecs, Pecs, \\ Baranya, Hungary \\ ${ }^{7}$ Tumour Biology, National Korányi Institute of Pulmonology, Budapest, \\ Hungary \\ ${ }^{8}$ Pulmonology, Semmelweis University, Budapest, Hungary
}

Acknowledgements The authors would like to thank Mary Keen, Emerita Professor of Pharmacology, University of Birmingham and Honorary Professor, University of Pecs for language editing.

Contributors All authors contributed to the study design and data analysis, and reviewed and approved the manuscript.
Funding This work was supported in part by the University of Pecs KA Research Fund 2018 (to JEP); GINOP-2.3.2.-15-2016-00022, TUDF0/51757-1/2019-ITM and by Humeltis.

Competing interests AS and JM-R: employees of Humeltis. JEP: received a grant and personal payments from Humeltis.

Patient consent for publication Not required.

Ethics approval The project was approved by the Ethical Committee of the University of Pecs (2014-RIKEB-5329-EKK) and the Medical Research Council of Hungary (366/2015 (46945-1/2015/EKU).

Provenance and peer review Not commissioned; externally peer reviewed.

Open access This is an open access article distributed in accordance with the Creative Commons Attribution Non Commercial (CC BY-NC 4.0) license, which permits others to distribute, remix, adapt, build upon this work non-commercially, and license their derivative works on different terms, provided the original work is properly cited, appropriate credit is given, any changes made indicated, and the use is non-commercial. See: http://creativecommons.org/licenses/by-nc/4.0/.

ORCID iD

Judit E Pongracz http://orcid.org/0000-0002-0278-5556

\section{REFERENCES}

1 Jemal A, Siegel R, Ward E, et al. Cancer statistics, 2009. CA Cancer J Clin 2009;59:225-49.

2 Langer CJ. Roles of EGFR and KRAS mutations in the treatment of patients with non-small-cell lung cancer. P T 2011;36:263-79.

3 Pikor LA, Ramnarine VR, Lam S, et al. Genetic alterations defining NSCLC subtypes and their therapeutic implications. Lung Cancer 2013;82:179-89.

4 Eberhardt WEE, De Ruysscher D, Weder W, et al. ESMO clinical practice guidelines: lung and chest tumours. Ann Oncol 2015;26:1573-88.

5 Planchard D, Popat S, Kerr K, et al. Metastatic non-small cell lung cancer: ESMO clinical practice guidelines for diagnosis, treatment and follow-up. Ann Oncol 2018;29:iv192-237.

6 Leichsenring J, Horak P, Kreutzfeldt S, et al. Variant classification in precision oncology. Int J Cancer 2019;145:2996-3010.

7 Boot A, Huang MN, Ng AWT, et al. In-depth characterization of the cisplatin mutational signature in human cell lines and in esophageal and liver tumors. Genome Res 2018;28:654-65.

8 Daste A, Domblides C, Gross-Goupil M, et al. Immune checkpoint inhibitors and elderly people: A review. Eur J Cancer 2017;82:155-66.

9 Blom K, Nygren P, Larsson R, et al. Predictive value of ex vivo chemosensitivity assays for individualized cancer chemotherapy: a meta-analysis. SLAS Technol 2017;22:306-14.

10 Breslin S, O'Driscoll L. Three-dimensional cell culture: the missing link in drug discovery. Drug Discov Today 2013;18:240-9.

11 Deng X, Nakamura Y. Cancer precision medicine: from cancer screening to drug selection and personalized immunotherapy. Trends Pharmacol Sci 2017;38:15-24.

12 Dean EJ, Ward T, Pinilla C, et al. A small molecule inhibitor of XIAP induces apoptosis and synergises with vinorelbine and cisplatin in NSCLC. Br J Cancer 2010;102:97-103.

13 Wang S, Xie J, Li J, et al. Cisplatin suppresses the growth and proliferation of breast and cervical cancer cell lines by inhibiting integrin 35 -mediated glycolysis. Am J Cancer Res 2016;6:1108-17.

14 Su WC, Chang SL, Chen TY, et al. Comparison of in vitro growthinhibitory activity of carboplatin and cisplatin on leukemic cells and hematopoietic progenitors: the myelosuppressive activity of carboplatin may be greater than its antileukemic effect. Jpn J Clin Oncol 2000;30:562-7.

15 Biziota E, Briasoulis E, Mavroeidis L, et al. Cellular and molecular effects of metronomic vinorelbine and 4-O-deacetylvinorelbine on human umbilical vein endothelial cells. Anticancer Drugs 2016;27:216-24.

16 Wouters A, Pauwels B, Lardon F, et al. In vitro study on the schedule-dependency of the interaction between pemetrexed, gemcitabine and irradiation in non-small cell lung cancer and head and neck cancer cells. BMC Cancer 2010;10:441.

17 Zasadil LM, Andersen KA, Yeum D, et al. Cytotoxicity of paclitaxel in breast cancer is due to chromosome missegregation on multipolar spindles. Sci Transl Med 2014;6:229ra43.

18 Chin TM, Quinlan MP, Singh A, et al. Reduced erlotinib sensitivity of epidermal growth factor receptor-mutant non-small cell lung cancer following cisplatin exposure: a cell culture model of second-line erlotinib treatment. Clin Cancer Res 2008;14:6867-76. 
19 Ono M, Hirata A, Kometani T, et al. Sensitivity to gefitinib (Iressa, ZD1839) in non-small cell lung cancer cell lines correlates with dependence on the epidermal growth factor (EGF) receptor/ extracellular signal-regulated kinase 1/2 and EGF receptor/Akt pathway for proliferation. Mol Cancer Ther 2004;3:465-72.

20 Schwartz LH, Litière $S$, de Vries $E$, et al. RECIST 1.1-Update and clarification: from the RECIST Committee. Eur J Cancer 2016;62:132-7.

21 The White House. The precision medicine initiative: data-driven treatments as unique as your own body. Available: https:// obamawhitehouse.archives.gov/blog/2015/01/30/precision- medicine-initiative-data-driven-treatments-unique-your-own-body [Accessed 5 Mar 2020].

22 Majumder B, Baraneedharan U, Thiyagarajan S, et al. Predicting clinical response to anticancer drugs using an ex vivo platform that captures tumour heterogeneity. Nat Commun 2015;6:1-14.

23 Hanahan D, Weinberg RA. Hallmarks of cancer: the next generation. Cell 2011;144:646-74.

24 Bohannon J. Fears of an Al pioneer: Stuart Russell argues that $\mathrm{Al}$ is as dangerous as nuclear weapons. Science 2015;349:252.

25 Giang I, Boland EL, Poon GMK. Prodrug applications for targeted cancer therapy. AAPS J 2014;16:899-913. 